\title{
Collisional Electron Detachment of Atomic Anions by Noble Gases: Universal Behavior at Intermediate Velocities
}

\author{
F. Zappa, L.F.S. Coelho, Ginette Jalbert, A. B. Rocha, S.D. Magalhães, and N.V. de Castro Faria \\ Instituto de Física, Universidade Federal do Rio de Janeiro, Caixa Postal 68528, Rio de Janeiro, 21941-972, RJ, Brazil
}

Received on 29 September, 2003

\begin{abstract}
Anions with atomic number lower than 18 had their total electron detachment cross sections measured in the 0.3-1.5 a.u. velocity range (40 keV-1.4 MeV) for $\mathrm{He}, \mathrm{Ne}$, and Ar targets with a experimental method developed in our laboratory. Several universal trends were observed: (a) each cross section can be described as the product of a target-dependent curve by a projectile-dependent constant; (b) for a given target all cross sections present a maximum at almost the same velocity; (c) the cross section ratios taken between different periods in a given column are target-independent.
\end{abstract}

Negative ions have fascinated atomic physicists since the pioneering works of J.J. Thomson in the beginning of the twentieth century. They present a variety of properties not usually found in their neutral and positive counterparts[1], utmost among them is their strong electron correlation effects. Concerning their structures, they usually possess only one bound state. The long standing interest caused by these and other unique properties nevertheless ran counter both to the complexity of the theoretical analysis of their structure and collisions and the relative scarcity of experimental installations for their study. The recent discovery of stable alkaline-earth anions is an example of these obstacles, as not only refined measurements were required for their detection but also most of the previous calculations, not fully considering correlation and relativistic effects, were unable to predict the very existence of these anions[2].

A similar picture arises in relation to studies of anionic collisions, which present more difficulties than the ones involving their positive counterparts[3, 4]. Even experimental studies of anion-gas collisions is still hampered by the limited availability of appropriate apparatuses, leading to scant measurements at the intermediate and high velocity regions[5,6]. In fact, though negative ion sources are now easily available, they are usually located at the first stage entrance of tandem accelerators dedicated to produce fast positive ion beams. Workable anion beams may of course be directly produced by placing negative ion sources at the negative high-voltage terminal of single-ended accelerators, but such set-ups are employed only at low velocities. Anion beams at intermediate and high velocities are then obtained from positive ion beams by electron capture or molecular fragmentation, with experimental inconveniences such as the limited choice of anions and the small currents obtained.

An innovative method to study such collisions, using a negative ion source and a tandem accelerator, has been recently developed in our laboratory $[7,8,9,10]$. This method employs the tandem gas stripper as a non-conventional target and allows measuring with ease absolute values of total cross sections at intermediate velocities, both for atomic anions electron detachment and for molecular anion destruction. Its essence lies on using the well known hydrogen charge-changing cross sections $[11,12]$ in order to make a correspondence between the stripper gas pressure and the pressure at the grounded high energy end of the accelerator, and in this way circumventing the need to place a pressure gauge at the tandem high voltage terminal. Several characteristics turn our method into a very powerful one for systematic studies of anion collisions. One is the external feeding of the target gas, allowing the easy study of different gases. Also, with modern tandem accelerators we can have in the first accelerator stage energies as low as $40 \mathrm{keV}$ and as high as several $\mathrm{MeV}$ and also a high beam current stability that simplifies the normalization procedure. Finally, the fact that the negative ions leave the accelerator always with the same small initial energy, irrespective of the terminal voltage, because they are accelerate in the first stage and desaccelerate in the second.

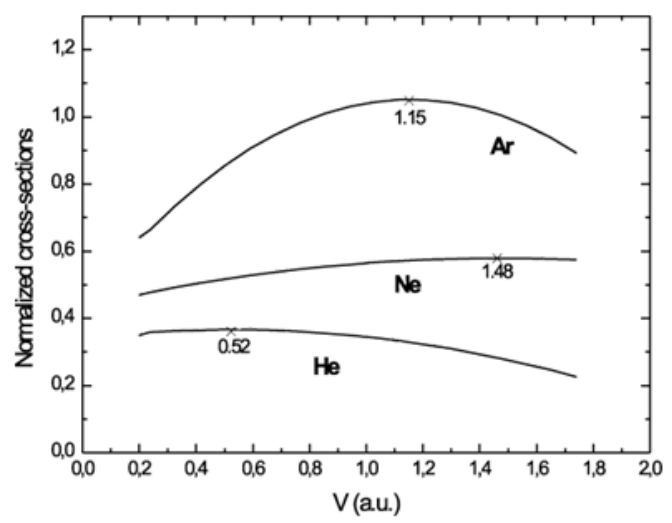

Figure 1. A polynomial fit to the normalized total electron detachment cross sections for several anions incident on (a) $\mathrm{He}$, (b) $\mathrm{Ne}$ and (c) Ar targets, as functions of the relative velocity in atomic units. The fluorine cross section was taken as the standard. 
The first study undertaken with our method was the collision of atomic anions with $\mathrm{n} p^{3}$ configurations $\left(\mathrm{C}^{-}, \mathrm{Si}^{-}\right.$, and $\mathrm{Ge}^{-}$) with $\mathrm{He}, \mathrm{Ne}$ and $\operatorname{Ar}[7,8]$. Several very interesting results were then obtained: (a) the cross sections for each target had almost the same velocity dependence, (b) target-independent factors scaled all the data in three curves, one for each gas, and (c) these factors increased as the electron affinity increased, i.e. the easiness to detach electrons from these anions increased when their binding energies increased. Besides these results, each cross section curve had a conspicuous maximum $\sigma_{m}$ at a projectile-independent velocity $\mathrm{v}_{m}$. Similar properties had already been observed by Andersen et al[6] for alkali atomic anions $\left(\mathrm{H}^{-}, \mathrm{Li}^{-}, \mathrm{Na}^{-}\right.$, and $\mathrm{K}^{-}$) colliding with noble gases, except that the cross sections scaled as the inverse square of the electron affinity. As further indications that the transitional behavior is quite independent of the anionic projectile, similar scalings were also verified for $\mathrm{C}_{n}^{-}(\mathrm{n}=2-4)$ and $\mathrm{Si}_{n}^{-}(\mathrm{n}=2-4)$ cluster anions colliding with noble gases(Zappa et al[9]), and even for more complex systems such as $\mathrm{B}_{n}^{-}(\mathrm{n}=1,2)$ and $\mathrm{Al}_{n}^{-}(\mathrm{n}=1-4)$ colliding with $\mathrm{N}_{2}[10]$

One could therefore suggest that the scalings in anionatom collisions, and in particular the lack of clear dependencies on electron affinity, were accidental events as only $\mathrm{n} s^{2}$ and $\mathrm{n} p^{3}$ anions were studied and their electron affinities differed only by less than $10 \%$. In order to better understand these phenomena we have undertaken an extensive study of detachment cross sections, for atomic anions of the 2 nd and the 3rd rows of the Periodic Table of Elements in collisions with noble gases. The projectiles under study had several configurations $-\mathrm{n} p^{2}\left(\mathrm{~B}^{-}\right.$and $\left.\mathrm{Al}^{-}\right), \mathrm{n} p^{5}\left(\mathrm{O}^{-}\right.$and $\left.\mathrm{S}^{-}\right)$, and $\mathrm{n} p^{6}\left(\mathrm{~F}^{-}\right.$and $\left.\mathrm{Cl}^{-}\right)-$and these results were compared with our previous $\mathrm{n} p^{3}$ results. We also measured $\mathrm{Li}^{-}$detachment, and compared with the Andersen et al previous $\mathrm{n} s^{2}$ results[6].

These systematic experiments were performed at the "Instituto de Física da Universidade Federal do Rio de Janeiro" and only a brief description of the experimental setup will be given here, as it has been previously described[7]. Anions of all the studied elements were produced in the cesium-sputtering ion source of the $1.7 \mathrm{MV}$ tandem accelerator 5SDH (NEC). The stripper was taken as a differentially pumped gas cell (without window), $1 \mathrm{~cm}$ wide and $47 \mathrm{~cm}$ long, placed between the two accelerator tubes. Two 500liter/sec $\left(\mathrm{N}_{2}\right)$ turbomolecular pumps were located at each extremity of the tandem, providing a residual pressure of $10^{-8}$ Torr. After exiting the tandem the anions were magnetically analyzed and detected by a Faraday cup placed at the 15 degrees exit of the magnet. The total (and absolute) detachment cross sections were extracted from exponential current decay curves, obtained by varying the target pressure and recording the Faraday cup current. Uncertainties in the exponential fitting procedure and small fluctuations in the initial ion beam currents were the main causes of uncertainty in the measured cross sections, estimated to be less than $5 \%$.

Our results showed again the remarkable feature that the cross sections for a given target had maxima at almost the same velocity, irrespective of the projectile. This suggested that the cross sections could be factored in a product of some target-dependent function, containing all the velocity dependence, and a constant which would give the over-all magnitude. In order to isolate each contribution we performed a normalization of the cross section values to the respective $\mathrm{F}^{-}$cross section, for each gas, using a least square fit procedure. A second order polynomial curve was fit to the normalized cross section values and the result is shown in Fig. 1 , with the normalization constants $\mathrm{K}$ being given on Table I. The absolute values of the presently measured cross sections will be presented in a forthcoming article. In Table I, and on the following analysis, besides our present data we included our $\mathrm{n} p^{3}$ previous results $[7,8]$ and the Andersen $e t$ al[6] data for the alkaline atoms. Table I also presents the values of the anion electron affinity and the parent atom ionization potential.

What we see in Table $I$ is that the normalization factors for a given $\mathrm{n} p^{m}$ projectile are quite similar, irrespective of the target, the neon data being slightly smaller than helium and argon data. This is quite surprising since it would mean that all the target contribution would be contained in the velocity-dependent function. Also intriguing is the absence of any clear overall dependence on the electron affinity: while the $\mathrm{n} s^{2}$ anion cross sections increase as the electron affinity decreases, a fact already verified by Andersen $e t$ al[6], the situation is exactly the reverse for $\mathrm{n} p^{m}$ anions of any given Periodic Table group (i.e. at any given $m$ value).

We can also see from Table I that for a given period the factors tend to decrease as one goes to the right of the Periodic Table and going down any fixed group the factors always increase. An alternative way of seeing this is Table II, where we divide the scaling factors of the 3rd period anions by the corresponding ones of the 2 nd period in the same group. We should point out that, although projectile electron affinities vary by as much as a factor of 15 (and ionization potentials by a factor of 3 ) and three distinct targets are being employed, the fractions of Table II present no target dependence (not even for neon) and differ at most by $20 \%$.

Concerning the velocity behavior, we can observe in Fig. 1 that there is a non-monotonic dependence on the target atomic numbers or, in other words, the maxima of $\mathrm{Ne}$ appear at a much higher velocity than for $\mathrm{Ar}$ and $\mathrm{He}$. In a previous article [8] we tried to interpret the position of these maxima as a consequence of the slope of the cross sections in the low velocity region, particularly near the threshold. In this region a quasimolecular model would apply. In this model, there is a crossing point between the quasimolecular potential energy curves asymptotically corresponding to (a) two neutral atoms and (b) one neutral atom and the anion. This crossing point corresponds to a maximum in the probability for detachment. Olson and Liu[13] made calculations for $\mathrm{H}^{-}$colliding with $\mathrm{He}, \mathrm{Ne}$ and $\mathrm{Ar}$. The present data indicate that this fenomenon should happen not only for $\mathrm{H}^{-}$but for all the other projectiles. Motivated by these experimental observations, we are doing calculations using the MollerPlesset method up to second order (MP2) with gaussian basis set. A preliminary result indicated that in all considered cases the crossing point is localized about the same internuclear distance. As He penetrate deeper in the electronic 
cloud of any particular anion when compared to $\mathrm{Ne}$ and $\mathrm{Ar}$, due to the number of electrons, its inter-electronic repulsion is smaller. As a consequence, the enhancement of the electron detachment by $\mathrm{He}$ with respect to $\mathrm{Ne}$ and Ar makes its detachment cross section larger near the threshold, pulling its cross section maximum to the origin. The fact that the velocity maximum position of $\mathrm{Ne}$ is greater than the one of Ar is more difficult to explain with simple arguments.

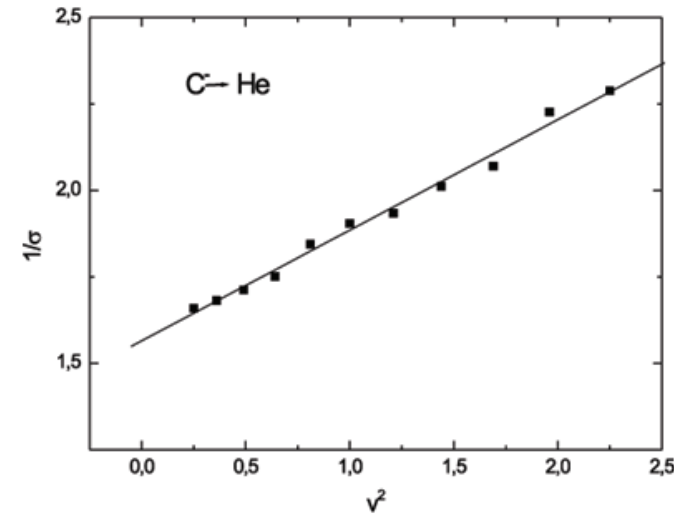

Figure 2. A typical example of the linear relation between the inverse of the cross section and the square of the velocity.

In the high velocity regime a simplified version of the Born Approximation[14] gives in general a good description of the detachment process. An analytical version developed by Meron and Johnson[15] was modified and applied in reference[16]. The most interesting result of this modification was the prediction of a linear relation between the inverse of the cross section and the square of the velocity. A typical example of this experimental dependence is shown in Fig. 2. In fact, in all considered cases of the present work, this linear relation was observed for velocities higher than the ones corresponding to the maximum. Thus not only the model applies well to high velocities, but we could also show from the parameters extracted from the linear fit that the empirically found factorization of the cross sections, into a target and a projectile factors, using the functional velocity dependence (essentially determined by the target) is justified by the model.

In conclusion, the striking characteristics concerning scalings and other common properties in the detachment process at intermediate velocities for $\mathrm{np}^{3}$ anions colliding with noble gases, were confirmed in a more general study for anions with other $\mathrm{n} p^{m}$ configurations. Considering the wide choice of configurations of the anions under study, with electron affinity values going from 0.277 to $3.613 \mathrm{eV}$ and atomic numbers going from 1 to 17 , the existence of such universal set of characteristics is striking. A simplified version of the Born Approximation, for the high velocity regime, and a preliminary calculation in the framework of a quasi-molecular approach, for the low velocity regime, explain these facts.
TABLE 1. Cross section normalization constants $K$, relative to $\mathrm{F}^{-}$. Includes previous data, ${ }^{*}[8]$ and ${ }^{* *}[6]$, electron affinities, ${ }^{+}[1]$, and ionization potentials.

\begin{tabular}{|c|c|c|c|c|c|c|}
\hline \multicolumn{2}{|c|}{ Projectile } & \multicolumn{3}{|c|}{ Target } & $\mathrm{EA}^{+}$ & $\mathrm{I}$ \\
\hline Group & Anion & $\mathrm{He}$ & $\mathrm{Ne}$ & $\mathrm{Ar}$ & $(\mathrm{eV})$ & $(\mathrm{eV})$ \\
\hline $2 \mathrm{~s}^{2}$ & $\mathrm{Li}^{-}$ & 2.20 & 1.47 & 2.41 & 0.618 & 5.36 \\
\hline $3 \mathrm{~s}^{2}$ & $\mathrm{Na}^{-*}$ & 2.82 & 1.89 & 3.10 & 0.548 & 5.14 \\
\hline $2 \mathrm{p}^{2}$ & $\mathrm{~B}^{-}$ & 1.53 & 1.43 & 1.50 & 0.280 & 8.26 \\
\hline $3 \mathrm{p}^{2}$ & $\mathrm{Al}^{-}$ & 2.08 & 2.02 & 2.19 & 0.433 & 5.96 \\
\hline $2 \mathrm{p}^{3}$ & $\mathrm{C}^{-* *}$ & 1.61 & 1.40 & 1.69 & 1.262 & 11.22 \\
\hline $3 \mathrm{p}^{3}$ & $\mathrm{Si}^{-* *}$ & 2.06 & 1.80 & 2.02 & 1.389 & 8.12 \\
\hline $2 \mathrm{p}^{5}$ & $\mathrm{O}^{-}$ & 1.19 & 1.10 & 1.17 & 1.461 & 13.55 \\
\hline $3 \mathrm{p}^{5}$ & $\mathrm{~S}^{-}$ & 1.89 & 1.70 & 1.87 & 2.077 & 10.3 \\
\hline $2 \mathrm{p}^{6}$ & $\mathrm{~F}^{-}$ & 1.00 & 1.00 & 1.00 & 3.401 & 17.34 \\
\hline $3 \mathrm{p}^{6}$ & $\mathrm{Cl}^{-}$ & 1.60 & 1.55 & 1.61 & 3.613 & 12.95 \\
\hline
\end{tabular}

TABLE 2. Cross section ratio $k=\sigma\left(3^{r d}\right.$ row $) / \sigma\left(2^{\text {nd }}\right.$ row $)$ for anions in the same group of the Periodic Table, and $\mathrm{He}, \mathrm{Ne}$ and $\mathrm{Ar}$ targets. $\left(^{*}:[6]\right)$.

\begin{tabular}{|c|c|c|c|c|}
\hline \multirow{2}{*}{$\begin{array}{c}\text { Cross section } \\
\text { ratios }\end{array}$} & \multicolumn{4}{|c|}{$k$} \\
\cline { 2 - 5 } & $\mathrm{He}$ & $\mathrm{Ne}$ & $\mathrm{Ar}$ & average \\
\hline $\mathrm{n} s^{2} \mathrm{Na} / \mathrm{Li}^{*}$ & 1.28 & 1.28 & 1.28 & 1.28 \\
\hline $\mathrm{n} p^{2} \mathrm{Al} / \mathrm{B}$ & 1.36 & 1.41 & 1.46 & 1.41 \\
\hline $\mathrm{n} p^{3} \mathrm{Si} / \mathrm{C}$ & 1.28 & 1.28 & 1.30 & 1.29 \\
\hline $\mathrm{n} p^{5} \mathrm{~S} / \mathrm{O}$ & 1.59 & 1.55 & 1.60 & 1.58 \\
\hline $\mathrm{n} p^{6} \mathrm{Cl} / \mathrm{F}$ & 1.60 & 1.55 & 1.61 & 1.59 \\
\hline
\end{tabular}

\section{Acknowledgments}

This work was partially supported by the Brazilian agencies CNPq, FUJB and FAPERJ.

\section{References}

[1] T. Andersen, H.K. Haugen, and H. Hotop, J. Phys. Chem. Ref. Data 28, 1511 (1999).

[2] T. Andersen, H.H. Andersen, P. Balling, P. Kristensen and V.V. Petrunin, J. Phys. B: At. Mol. Opt. Phys. 30, 3317 (1997).

[3] J.M. Rost, Phys. Rev. Letters 82, 1652 (1999).

[4] F. Robicheaux, Phys. Rev. Letters 82, 707 (1999).

[5] W.J. Lichtenberg, K. Bethge, and H. Schmidt-Böcking, J. Phys. B: Atom. Molec. Phys. 13,343(1980).

[6] N. Andersen, T. Andersen, L. Jepsen, and J. Macek, J. Phys. B: At. Mol. Phys. 17, 2281 (1984).

[7] H. Luna, S.D. Magalhães, J.C. Acquadro, M.H.P. Martins, W.M.S. Santos, G. Jalbert, L.F.S. Coelho, and N.V. de Castro Faria, Phys. Rev. A 63, 022705 (2001). 
[8] H. Luna, F. Zappa, M.H.P. Martins, S.D. Magalhães, G. Jalbert, L.F.S. Coelho, and N.V. de Castro Faria, Phys. Rev. A. 63, 052716 (2001)

[9] F. Zappa, L.F.S. Coelho, S.D. Magalhães, J. C. Acquadro, Tania S. Cabral, Ginette Jalbert and N.V. de Castro Faria, Phys. Rev. A. 64, 032701 (2001).

[10] F. Zappa, L.F.S. Coelho, S.D. Magalhães, W.M.S. Santos, A.M. Luiz, M.H.P. Martins, A.L.F. de Barros, J.A.M. Pereira, and N.V. de Castro Faria, Phys. Rev. A. 67, 012702 (2003).

[11] Y. Nakai, T. Shirai, T. Tabata, and R. Ito, Atom. Data and Nucl. Data Tables 37, 69 (1987).
[12] D.P. Almeida, N.V. de Castro Faria, F.L. Freire Jr., E.C. Montenegro, and A.G. de Pinho, Phys Rev. A36, 16 (1987).

[13] R.E. Olson and B.Liu, Phys Rev. A17, 1568 (1978); Phys Rev. A20, 1344 (1979) and Phys Rev. A22, 1389 (1980).

[14] D.P. Dewangan and H.R.J. Walters, J. Phys. B 11, 3983 (1978)

[15] M. Meron and B.M, Johnson, Phys. Rev. A41, 1365 (1990).

[16] W. Wolff, L.F.S. Coelho, H.E.Wolf, and N.V. de Castro Faria, Phys. Rev. A 45, 2978 (1992). 